\title{
EFFECT OF FILM FORMATION METHOD AND ANNEALING ON CRYSTALLINITY OF POLY(L-LACTIC ACID) FILMS
}

\author{
Shan-Ting Hsu, Y. Lawrence Yao \\ Department of Mechanical Engineering, Columbia University \\ New York, NY, USA
}

\begin{abstract}
Poly(L-lactic acid) (PLLA) has been shown to have potential medical usage such as in drug delivery because it can degrade into bioabsorbable products in physiological environments, and its degradation is affected by crystallinity. In this paper, the effect of film formation method and annealing on the crystallinity of PLLA are investigated. The films are made through solvent casting and spin coating methods, and subsequent annealing is conducted. The resulting crystalline morphology, structure, conformation, and intermolecular interaction are examined using optical microscopy, X-ray diffraction, and Fourier transform infrared spectroscopy. It is observed that solvent casting produces category 1 spherulites while annealed spin coated films leads to spherulites of category 2. Distinct lamellar structures and intermolecular interactions in the two kinds of films have been shown. The results enable better understanding of the crystallinity in PLLA, which is essential for its drug delivery application.
\end{abstract}

\section{INTRODUCTION}

There is a significant interest in use of biodegradable polymers due to their biocompatibility and biodegradability. Poly lactic acid (PLA) is a biodegradable polymer and can be obtained from renewable sources such as corn starch. It has a wide range of applications, such as in food packaging and tissue engineering. PLA is also of interest in drug delivery application because it can degrade into bioabsorbable products in physiological environments. Its degradation mainly comes from the cleavage of its ester groups, and is sensitive to chemical hydrolysis. In polymeric drug delivery system, drugs are encapsulated in polymer. As the polymer degrades, the drugs are released, and the release rate is determined by the polymer degradation rate. Crystallinity of polymer is a factor affecting the degradation rate. A good review on the effect of crystallinity on degradation and drug release can be found in [1]. In general, less crystallinity accelerates degradation and drug release rate. To create polymeric drug delivery system, drug molecules are dispersed or dissolved in the polymeric solution, formed through melting the polymer or dissolving it in a solvent. During the process the polymer may crystallize. The crystal structure also influences the degradation rate. Tsuji and Ikada [2] studied the hydrolysis of PLA films and proposed that it begins in the amorphous region between the crystalline lamellae, followed by the disorientation of the lamellae and disappearance of the spherulitic structure.
Accordingly, crystallinity in PLA films plays a significant role when in drug delivery system.

Efforts have been done to investigate the crystallization behavior and resulting crystal structures of PLA films. Among them, solvent casting [3,4] and spin coating [5] are the two commonly used film formation methods. In solvent casting, a quiescent polymer solution is deposited on a substrate, while evaporation of solvent leaves a solid polymer film. During the casting process crystallization from solution can occur if the solvent evaporation rate is low enough for polymer macromolecules to diffuse to the crystal growth front and overcome the energy barrier of deposition. Category 1 spherulites, defined by Norton and Keller [6], can be commonly observed in this case $[3,7,8]$. Spin coating generates polymer films with thinner and more uniform thickness. This process involves rapid rotation of a polymer solution, with the centrifugal force pushing it to flow radially outward, decreasing its thickness. The solvent evaporates simultaneously. This process typically finishes within several seconds or minutes. Due to the rapid evaporation, it is generally less likely for crystal structures to develop in this process.

To increase crystallinity in the as cast/coated PLA film, various annealing processes have been attempted. Since crystallization from the melt produces PLA films with reduced continuity [9], it is of interest to focus on annealing from the glassy state, as conducted in [3-5]. Solvent cast films may be covered by spherulites. Subsequent annealing increases their degree of crystallinity, but has no effect on morphology $[3,4]$. For amorphous material, such as the non-annealed spin coated film, crystallization from glassy state can generate crystal structures different from category 1 spherulites. One common feature is the nodule structure [10-12], the nanometer-sized crystalline particles developed during annealing from the glassy state [13]. As annealing time and/or temperature increases, nodules enlarge, and may develop into larger rod-like crystals [10]. It is also observed that nodules develop to lath-like lamellae through merging or lateral aggregation [11,12]. At larger scale, the sheaf-like structures can be observed [14], and category 2 spherulites [6] are developed [15]. Crystal structures developed from the glassy state thus show distinct features from those developed from solution.

In spite of the fact that different crystallization methods lead to different crystalline morphologies and structures, the effect of processing methods on its crystal structure has not been fully explored. Pluta and Galeski [9] conducted wide- 
angle X-ray diffraction (WAXD) measurements on PLA films crystallized from melt and glass, and peculiarity in the profiles for the latter is noticed. $\mathrm{Li}$ et al. [5] observed various PLA film morphologies crystallized from glass, while the crystal structure is not addressed. For annealed solvent cast PLA films, the crystal structures developed in the two crystallization steps are expected to be different, which has not drawn much attention. It is also worth considering the causes leading to the different spherulitic morphologies.

The primary objective of this study is to investigate the crystalline morphology and structure in PLA films formed through solvent casting and spin coating followed by annealing. To characterize these films, optical microscopy is used to observe the crystalline morphology. The crystal structure is studied through WAXD, and Fourier transform infrared (FTIR) is employed to investigate the possible intermolecular interaction and crystal structure on the molecular order.

\section{BACKGROUND}

\section{General properties and unit cell structure of PLA}

As a thermoplastic polymer, PLA has a glass transition temperature and melting temperature of around $55^{\circ} \mathrm{C}$ and $175^{\circ} \mathrm{C}$. It has three stereochemical forms: poly (L-lactic acid) (PLLA), poly (D-lactic acid) (PDLA), and poly (DL-lactic acid) (PDLLA), among which PLLA and PDLA are crystallizable. Depending on the preparation conditions, PLLA crystallizes in different forms: $\alpha, \beta$, and $\gamma$. The $\alpha$ form is more stable and can exist in solution cast and annealed samples. The unit cell structure of the $\alpha$ form is orthorhombic with two $10_{3}$ polymeric helices [16-18]. The lattice parameters are $\mathrm{a}=10.66 \AA, \mathrm{b}=6.16 \AA$, and $\mathrm{c}$ (chain axis) $=28.88 \AA$ [17], but the values may slightly change $[16,18]$, which can come from thermal expansion [19], chain compacting related to annealing temperature [20], fold surface irregularity [21], and internal stress associated with entanglement [22]. PLLA chains are folded in the $\langle 110\rangle$ direction in the orthorhombic unit cell [17,23], as illustrated in Figure 1.

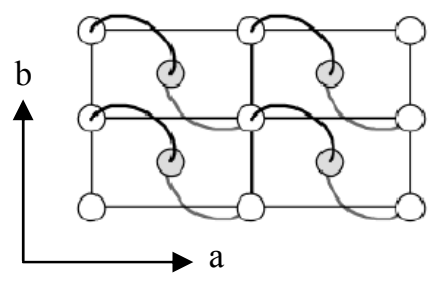

Figure 1 - Schematic representation of the chain-folding direction in PLLA crystals [23].

\section{Nucleation and crystallization}

Crystallization of polymers is composed of two steps: (1) radial growth of spherulites and (2) the crystallization after the spherulites impinge; the former is faster than the latter. To initiate crystallization, a stable primary nucleus with a critical size must be achieved so as to overcome the energy barrier of formation [24]

$$
\Delta G^{*}=\frac{k \sigma_{s}^{2} \sigma_{e}\left(T_{m}^{o}\right)^{2}}{\Delta h_{f}^{2}\left(T_{m}^{o}-T_{x}\right)^{2}}
$$

where $k$ is the geometric constant, $\sigma_{s}$ and $\sigma_{e}$ are the free energy for the sides and ends respectively, $T_{m}^{o}$ is the melting point of infinitely extended crystals, $\Delta h_{f}$ is the fusion heat, and $T_{x}$ is the crystallization temperature. Once the stable nucleus is formed, it provides a surface on which the amorphous molecule chain attaches crystallographically. It then folds back on itself and crystallize in a position adjacent to the first stem, as proposed by Hoffman et al for crystallization in unstrained melt or solution [25]. Assuming the deposition rate of each stem is identical, the rate can be expressed as [24]

$$
r=\frac{\eta_{0}}{L} \frac{k T}{h} e^{-\frac{\Delta f^{*}}{k T}} e^{-\frac{\Delta G_{1}}{2 k T}}\left(\frac{2 \sinh \frac{E}{2 k T}}{1+2 e^{-\frac{\Delta G_{1}}{2 k T} \sinh \frac{E}{2 k T}}}\right)
$$

where $\eta_{0}$ is the number of stems, $L$ the width of the growth front, $k$ the Boltzmann's constant, $T$ the temperature, $h$ the Planck constant, $\Delta G_{1}$ the free energy of depositing the first stem, $E$ the additional energy change as following stems are deposited. Since this model is applicable for crystallization from solution, it may account for the formation for the category 1 spherulites developed during casting as mentioned in Introduction.

For crystallization from the glassy state, a different route of crystallite growth has been observed, in which nano-sized nodules are developed first followed by coalescing with each other to form larger crystals such as lamellae [11]. The key issue of their coalescence is their ability to rotate, and the concurrent orientation adjustment of neighboring nodules is required for prefect coalescence. The coalescence can be opposed by chain entanglement, which gives a strong resistance to nodule rotation, and thus perfect alignment and coalescence cannot always be achieved. A similar route is proposed by Strobl for crystallization from the entangled melt [26]. In this model, granular crystals merge with one another and transform into a lamellar. The resulting lamellar is composed of mosaic block structures not perfectly aligned. This crystallization route also agrees with some experimental results for crystallization from the glassy state [27]. On this ground, the multi-step crystallization route can account for further annealing of the as cast/coated film from the glassy state and the resulting crystal structures.

\section{Structure of spherulites}

The spherulite is composed of crystalline lamellae and amorphous region. The third phase, the rigid amorphous phase (RAP), has also been discovered [28], and this 3-phase model has increasingly drawn attention. The RAP origins from the part of molecular chains trapped by the lamellae, which makes it immobile and remain vitrified even above the glass transition temperature.

In terms of architectures, Norton and Keller [6] divided the spherulites into two categories. The cause of different categories arises from the competition between the order in the crystal structure and the disorder of the crystallographic orientation; the latter is associated with the randomization of 
secondary nucleation occurring at the growth surface [29]. Because of random orientation of the secondary nucleation, the original crystalline order is disrupt so as to achieve isotropy. For category 1 spherulites, disrupt occurs in the early stage, and thus multiple directional growth is achieved in the beginning of their development. The disorder disrupts the crystalline anisotropy later for category 2 spherulites, leading to the threadlike structure at the center and sheaf outside.

The origin of disorder during the secondary nucleation can occur via three mechanisms [29]. The first is caused by static spatial heterogeneity such as foreign particles, which deflect the tip of growing lamellae and perturb their growth direction. The second is due to the small rotational diffusion coefficient compared with the translational diffusion coefficient. The molecule thus has difficulty changing orientation when depositing on the growth front and thus causes disorder. The third is due to the non-crystallographic branching, which may occur at a short distance away from the mother lamellae [30]. This gives evidence that it can be a result of the secondary nuclei growth, which are formed by the loose loop or protruding cilia of molecules trapped in the mother lamellae. According to simulation results [29], the third mechanism dominates in most cases.

\section{MATERIALS AND METHODS}

\section{Sample preparation and annealing}

PLLA granule samples were kindly provided by PURAC Netherlands and were used as received. The inherent viscosity $(\eta)$ in chloroform at $25^{\circ} \mathrm{C}$ is $1.6 \mathrm{dl} / \mathrm{g}$. The PLLA granules were dissolved in methylene chloride $\left(\mathrm{CH}_{2} \mathrm{Cl}_{2}\right)$ from Sigma Aldrich, with solution concentration of $0.1 \mathrm{~g}$ PLLA $/ 3 \mathrm{~mL}$ $\mathrm{CH}_{2} \mathrm{Cl}_{2}$. The solution is stirred with a magnetic stirrer for 4 hours. Fifteen $\mathrm{mL}$ of the solution was cast in a covered Petri dish with a glass slide put on the bottom as a substrate. The solution was left to settle in a hood at $25^{\circ} \mathrm{C}$ for 24 hours. The film formed on the glass substrate was used as solvent cast samples. Its thickness, measured by the profilometer, is 20-25 $\mu \mathrm{m}$. The PLLA/ $\mathrm{CH}_{2} \mathrm{Cl}_{2}$ solution with doubled concentration was spin-coated on a glass substrate to produce films with thickness larger than $1 \mu \mathrm{m}$ so that film thickness effect can be eliminated [31]. Both concentrations for solvent casting and spin coating solutions are lower than the overlap concentration $\mathrm{c}^{*}$, which is between 0.6 and $2.3 \mathrm{~g} / \mathrm{ml}$ as calculated by [32]

$$
\frac{\frac{\mathrm{M}}{\mathrm{N}_{\mathrm{A}}}}{\frac{4}{3} \pi \mathrm{R}_{\mathrm{g}}{ }^{3}} \leq \mathrm{c}^{*} \leq \frac{\frac{\mathrm{M}}{\mathrm{N}_{\mathrm{A}}}}{\mathrm{R}_{\mathrm{g}}{ }^{3}}
$$

where $R_{g}, M$ and $N_{A}$ are the spherical region of radius, molecular weight and Avogadro constant, respectively. Thus, in both solutions the polymer molecules do not overlap with each other, and the effect of concentration may not influence the results. The rotational acceleration for spinning is 255 $\mathrm{rev} / \mathrm{min}^{2}$ and the rotational velocity is $1000 \mathrm{rpm}$. The film thickness is around $2.5 \mu \mathrm{m}$. The solvent cast and spin coated films were annealed in a furnace at $80^{\circ} \mathrm{C}, 110^{\circ} \mathrm{C}$, and $140^{\circ} \mathrm{C}$ for 1 and 3 hours; some spin coated films are annealed at $140^{\circ} \mathrm{C}$ for
8 and 24 hours to investigate effect of annealing time. The annealed films were subsequently quenched in room temperature to prevent further development of crystallites.

\section{Characterization}

The crystallinity in PLLA films was observed with an optical microscope (Olympus BX60) in the transmission mode. WAXD was accomplished using the Inel X-ray diffractometer. Film specimens were exposed to the monochromatic $\mathrm{CuK} \alpha$ radiation with wavelength $\lambda=0.15418 \mathrm{~nm}$ at $40 \mathrm{kV}$ and $30 \mathrm{~mA}$. The scattered intensity was measure at the Bragg angle (20) ranged from 5 to 90 degrees. Care was taken to adjust the incident angle of x-ray to prevent penetration into the substrate. A Perkin Elmer Spectrum 400 FTIR spectrometer with attenuated total reflectance (ATR) attachment using $\mathrm{ZnSe}$ crystal was used to measure the IR spectrum; the data were recorded with a $4 \mathrm{~cm}^{-1}$ resolution. The films were peeled from the glass substrate when measured.

\section{RESULTS AND DISCUSSION}

Different morphological features are observed in annealed solvent cast and spin coated films, as given in Figures 2(a) and 2(b). The crystal structures in the two films are also different from WAXD and FTIR measurements. Morphology, WAXD, and FTIR results will be discussed in the following sections.

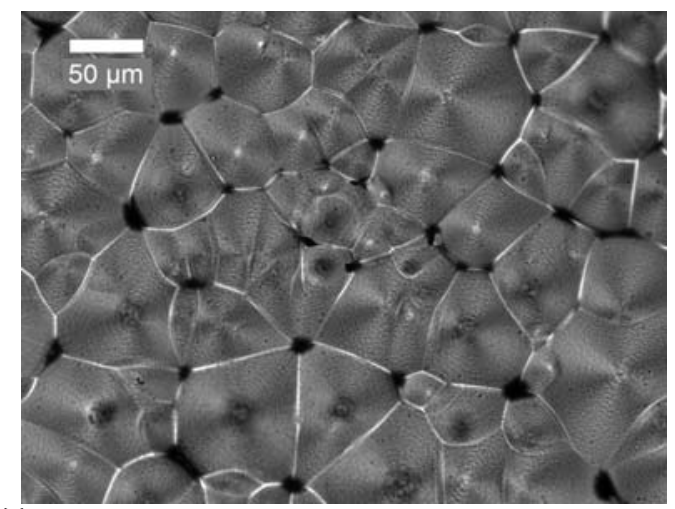

Figure 2(a) - The spherulitic structure in the solvent cast film annealed at $140^{\circ} \mathrm{C}$ for 3 hours. The spherulitic structure is similar to that in the as cast film.

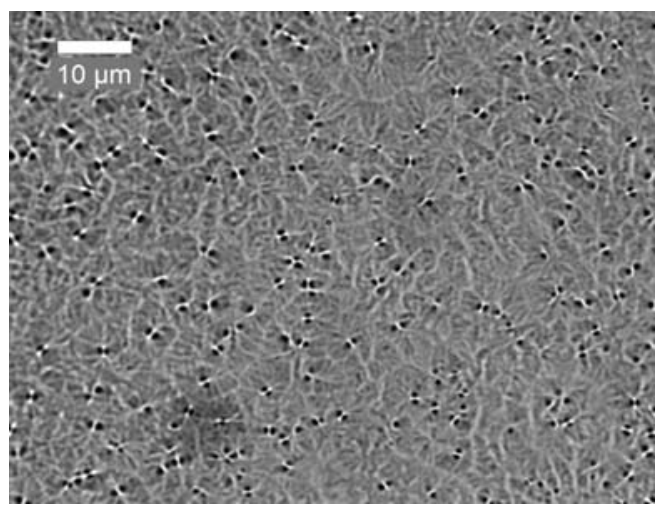

Figure 2(b) - The surface morphology of the thick spin coated film annealed at $140^{\circ} \mathrm{C}$ for 3 hours. The eye structure is observed. 


\section{Crystalline morphology}

Before annealing, spherulites already appear and impinge in the solvent cast films because the solvent evaporates at a rate low enough for crystals to grow. However, no observable feature exists in spin coated films. After annealing, the morphology in solvent cast films remains the same, while that in spin coated films changes significantly.

It is observed that in the annealed spin coated films, there are small black dots appearing in pairs with a bright threadlike "fiber" in between. This structure is similar to category 2 spherulites. The dots are the amorphous region known as the "eye structure" and the fiber is the stack of lamellae developing in a specific direction at the core of the spherulite. The spherulites formed in the solvent cast films are closer to category 1 , in which lamellae grow radially from the center and branch uniformly within the spherulite. It is proposed [29] that the order of the secondary nucleation at the crystal growth surface determines the structure difference: if disorder disrupts the orientation of crystal growth early, isotropic growth could occur at the center, leading to category 1 spherulite, and noncrystallographic branching is a dominant mechanism causing disorder [29].

The cause of different branching behaviors in the two films could be related to the different pressure gradients and stress fields between the branching and non-crystalline region induced by crystallization [24,33]. Since the crystalline region has a higher density and thus a smaller specific volume, its volume shrinks when crystallizing, and induces a pressure gradient causing a flow of surrounding material to compensate the volume shrinkage. This flow supplies polymer molecules to the branching growth surface, as in the case of solvent cast films, so that branching could easily happen and disturb the anisotropy. For highly viscous polymer melt, as in the spin coated films, the polymer chains connecting crystalline and non-crystalline regions tend to move to the growth front as the surrounding material gets crystallized and shrinks. However, these connecting chains are entangled with adjacent molecules, and thus are extended toward the crystal growth front. In such case, the shrinkage is not compensated by the flow, but by the local extension of molecules to the front [24]. Therefore, the supplying material is not available, and the growth of branches is thus unfavorable, making the isotropic growth unable to be achieved in the early stage of growth. This explains why category 1 spherulites exist in solvent cast films while category 2 spherulites are developed in spin coated films.

\section{Crystal structure}

The WAXD profiles of solvent cast and spin coated films are given in Figures 3(a) and 3(b). For solvent cast films, the WAXD profiles show three stronger peaks, $(010),(110) /(200)$, and (203), while only two peaks, (110)/(200) and (203), exist for the spin coated films. The $(110) /(200)$ peak remains prominent in all crystalline samples, including the nonannealed solvent cast film with lower degree of crystallinity. (The degree of crystallinity will be discussed below; see Figure 7.) This suggests the crystal structure in the (110) and/or (200) direction keeps ordered during crystallization. Since in
PLLA crystals the chains are folded in the $<110>$ direction first, forming a crystal surface for the subsequent chains to attach $[17,23]$, the $(110) /(200)$ peak may mainly come from the order of the chain folding in the $<110>$ direction.

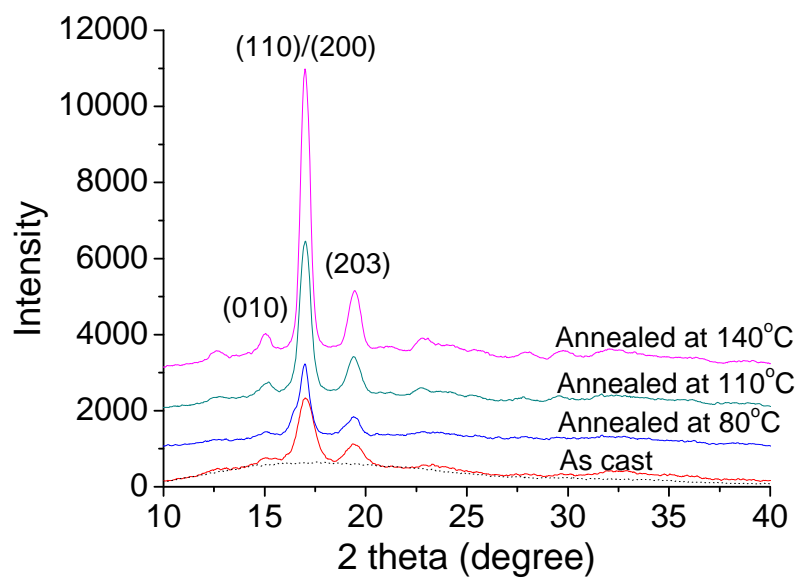

Figure 3(a) - XRD profiles for the solvent cast films annealed at different temperatures for 3 hours. The dot line under the as cast film is the fitted curve for the amorphous region.

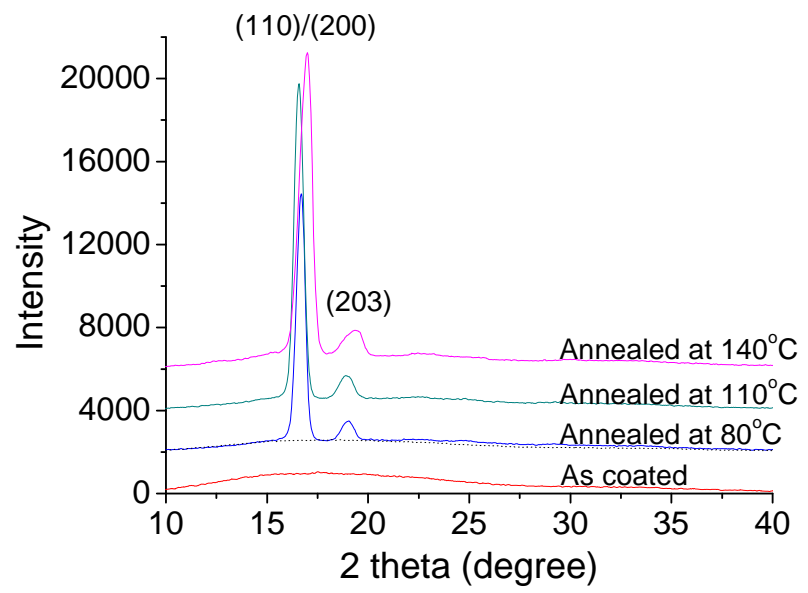

Figure 3(b) - XRD profiles for the spin coated films annealed at different temperatures for 3 hours. The dot line under the $80^{\circ} \mathrm{C}$ annealed film is the fitted curve for the amorphous region.

The (110)/(200) peaks gradually increase with the annealing temperature in solvent cast films, while for spin coated films the peak remains prominent even at lower annealing temperature. This is because the degree of crystallinity of spin coated films is always higher than that of solvent cast films under the same annealing conditions. The highly prominent peak in the spin coated films could also reflect that the crystallites are much more ordered in the chain folding direction when compared with other directions. We believe the reason is related to (1) the direction of chain folding and procedure of crystal growth, as discussed in the last paragraph, and (2) the possible nodular structure existing in the spin coated films. It is likely that the crystal structure annealed from the glassy state, as in the annealed spin coated 
films, is composed of nodular structures. The possible crystal structures in the solvent cast and annealed spin coated of films are thus sketched in Figures 4(a) and 4(b), respectively. This reduces the dimension in the crystal growth direction $\{110\}$ in each block, and in turn decreases the number of crystal planes in the $a$ and $b$ directions. Signal intensity may thus decrease. This explains the absence of the (010) peak and the decrease of the (203) peak. The fact that the (203) peak remains may be because the chain folding (depositing) length in a lamella, typically on the order of several hundred angstroms, is much larger than the displacement of (203) planes along the chain axis $(9.7 \AA)$. Therefore, enough planes still exist along this direction to produce a certain amount of diffraction.

When compared with crystallization from solution, which is how the spherulites form in solvent cast films, the molecular chains in spin coated film suffer less mobility. This makes them difficult to disentangle from their neighbors and get folded before depositing onto the near crystal growth surface. Under this circumstance, the fringed micellar crystallization can occur simultaneously, which roughens the folding surface when compared with uniformly folded crystal structure [34], as also indicated in Figures 4(a) and 4(b).

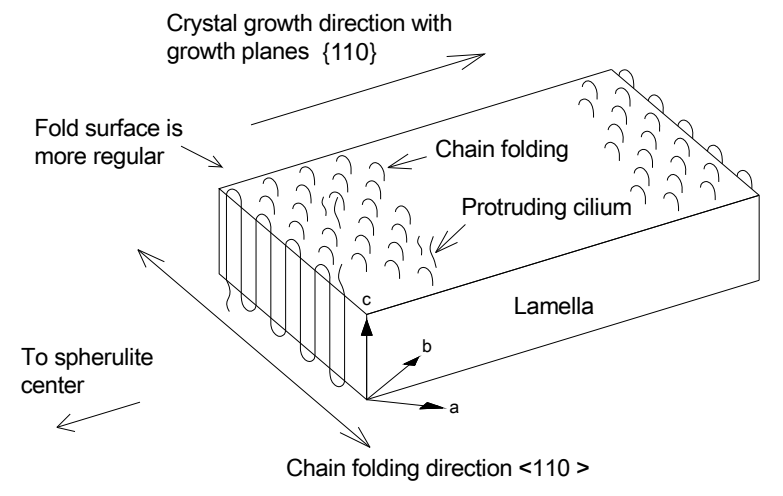

Figure 4(a) - Sketch of a possible crystal structure in the solvent cast films. The lateral sizes are on the order of $\mu \mathrm{m}$. The thickness is on the order of $\mathrm{nm}$.

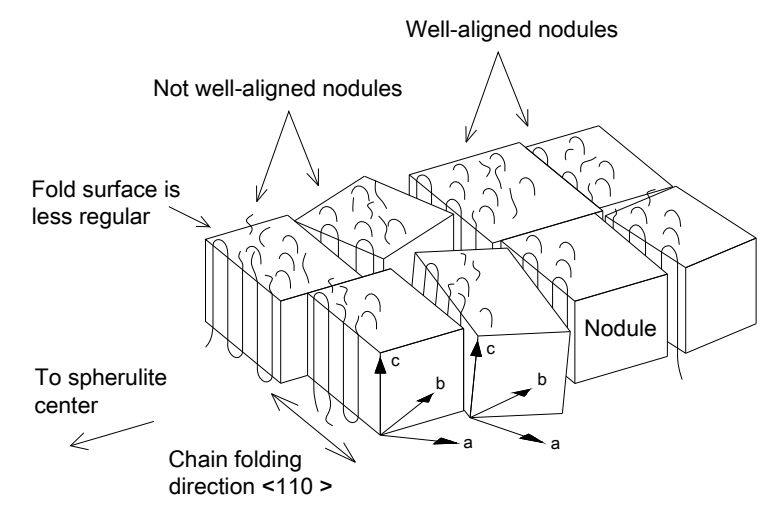

Figure 4(b) - Sketch of a possible crystal structure in the spin coated films. The lateral sizes are on the order of $\mu \mathrm{m}$. The thickness and nodule size are on the order of $\mathrm{nm}$.
The intensity ratio of the two strongest peaks, (110)/(200) and (203), is calculated based on the area under the peaks and is used to investigate the ordered structure developing in these directions. The results for 3-hour annealed films are given in Figure 5. It can be seen that the ratio for the spin coated films does not change with respect to annealing temperature, but that for the solvent cast films slightly increases with the annealing temperature. The same trend holds for the 1-hour annealing results. The similar ratio in spin coated films implies that similar crystal structures are developed. Solvent cast films always have smaller intensity ratios, which, based on the discussion above, is because of more uniform lamellae with more (203) reflection planes. The (203) peak can thus be more prominent, reducing the intensity ratio. In addition, it is noted that the ratio gradually increases with annealing temperature. Since the degree of crystallinity increases with annealing temperature, the newly formed crystal structure is believed to account for the increased peak intensity. This is because the crystal structure in solvent cast films during annealing is not formed in solution; instead, it forms in an environment more similar to that in spin coated films. Based on the discussion above, the newly formed crystallites may be in form of nodules, leading to a smaller (203) and a higher intensity ratio.

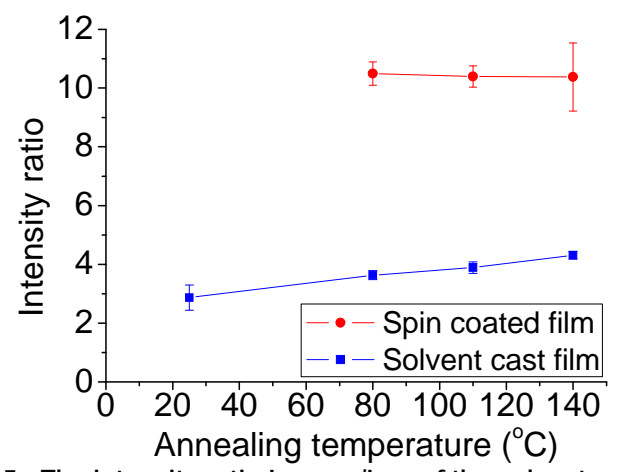

Figure 5 - The intensity ratio $I_{(110) /(200)} / I_{(203)}$ of the solvent cast films and spin coated films annealed for 3 hours. The error bar represents the standard deviation.

Chain compacting also differs in the two kinds of films, which is revealed by peak location ( $2 \theta$ value), given in Figure 6. It is noticed that the peak location for spin coated films is smaller than that for solvent cast films. From the Bragg's law, $\lambda=2 \mathrm{~d} \sin \theta$, solvent cast films have smaller $\mathrm{d}$-spacings and therefore a more ordered and compact crystal structure. This can originate from the effect of folds [21]: the irregular folding surfaces prevent compacting of chains, which results in the increase of the basal area of the unit cell, accordingly enlarging the lattice parameters $a$ and $b$, while $c$ remains unchanged. As discussed previously, the folding surface of the lamellae in solvent cast films should be more uniform, while that in the spin coated films is rougher, which leads to smaller lattice parameters $a$ and $b$ in solvent cast films. Based on the plane spacing equation for orthorhombic cells 


$$
\frac{1}{d^{2}}=\frac{h^{2}}{a^{2}}+\frac{k^{2}}{b^{2}}+\frac{l^{2}}{c^{2}}
$$

where $d$ is the $d$-spacing value, and $h, k$, and $l$ are the plane indices, smaller $a$ and $b$ result in smaller $d$, and therefore larger $2 \theta$ values are measured in the solvent cast films. Another reason of larger d-spacing in the spin coated films is the stress in the structure [22], which is induced by the centrifugal force caused during coating process [35]. Higher annealing temperature gives energy to the molecules to release the stress and to achieve equilibrium state [23]; it also enables molecular chains to change their conformations to that favors chain compacting. This explains the smallest $\mathrm{d}$-spacing for $140^{\circ} \mathrm{C}$ annealed spin coated film. We do not observe change of $2 \theta$ values in solvent cast films with annealing temperature. This may be due to the fact that the dominant spherulitic structure is formed during the same casting process, and the effect of annealing on structure formation is not as significant as that in the spin coated films.

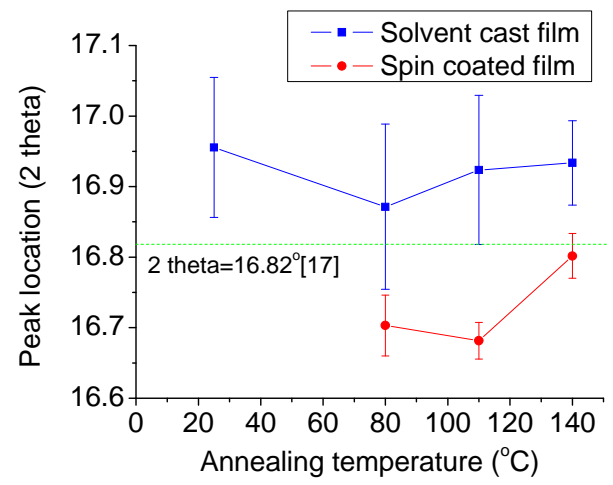

Figure 6 - The location of the (110)/(200) peak for the solvent cast and spin coated films annealed for 3 hours. The error bar represents the standard deviation.

Different $2 \theta$ values occur in solvent cast films and spin coated films. The $2 \theta$ value calculated from the lattice parameters proposed by Miyata and Masuko [17] is $16.82^{\circ}$. The values, although very close, have slight difference, which is a result of different processing and crystallization conditions, as discussed in the last paragraph, and again demonstrates that the lattice parameters of polymers can vary even for the same material.

Figure 7 gives the degree of crystallinity for 3-hour annealed films. The degree of crystallinity increases with annealing temperature, as expected. Similar results are obtained for the 1-hour annealed films, except that the degrees of crystallinity decrease by around $3 \%$ for the annealed spin coated films, while those for solvent cast films do not change, suggesting that 1 hour is enough to achieve highest secondary crystallization after the impingement of spherulites for solvent cast films. This secondary crystallization may be contributed to the amorphous chains within the lamellae, which represents material inherently slower crystallizing due to chain imperfection such as, impurities, chain branching, or atactic components [24], and higher temperature by annealing favors their crystallization.

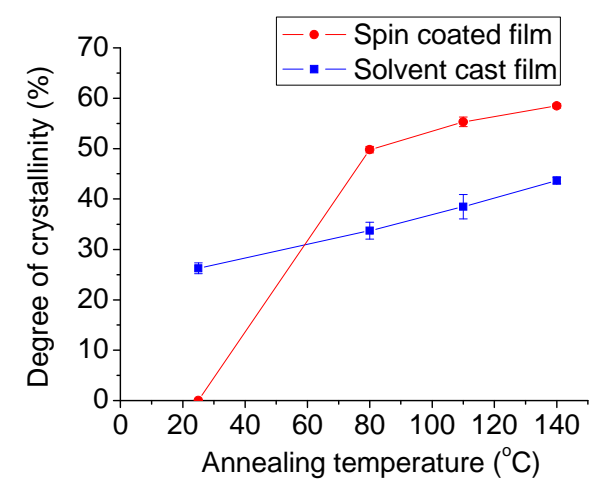

Figure 7 - The degree of crystallinity of the solvent cast and spin coated films annealed for 3 hours. Annealing temperature of $25^{\circ} \mathrm{C}$ means the as cast film. The error bar represents the standard deviation.

It is also noticed that the degree of crystallinity for solvent cast films is always lower than the spin coated films, which suggests that part of the structure in the as cast films can hardly crystallize. This can be explained by the structure in the spherulites. From optical microscope images, it is revealed that the non-annealed solvent cast film is already filled with spherulites; however, the degree of crystallinity is lower than the annealed spin coated films, in which the spherulites also fill the entire sample. This means that the spherulites in nonannealed solvent cast film are "open" when compared with the spherulites in spin coated films, and that more amorphous region is trapped within the spherulites [36]. The molecular chains in the trapped amorphous region, based on the 3-phase model, have higher possibility to be fixed by the lamellae surfaces, forming the rigid amorphous phase. Crystallization then becomes less likely due to its vitrification. This explains the phenomenon that the degree of crystallinity in solvent cast films is always lower.

\section{Conformation and intermolecular interaction}

The conformation and intermolecular interaction is studied through FTIR. The spectra of solvent cast and spin coated films annealed at different conditions are given in Figures 8(a) and $8(\mathrm{~b})$, respectively. The carbonyl $(\mathrm{C}=\mathrm{O})$ stretching region, $\mathrm{CH}_{3}$ and $\mathrm{CH}$ bending region, and the skeletal stretching region (C-O-C) show prominent peaks. Along the molecular chain of PLLA, there are three skeletal bonds: $\mathrm{C}-\mathrm{O}$ (ester), $\mathrm{O}-\mathrm{C}_{\alpha}$, and $\mathrm{C}_{\alpha}-\mathrm{C}$, in which the ester bond can be assumed trans because of increased stability due to $\mathrm{C}=\mathrm{O}$ double bond conjugation [37]. The $\mathrm{O}-\mathrm{C}_{\alpha}$ bond has two minimal energies if its bond rotation angle $\varphi$ is $-160^{\circ}$ and $-73^{\circ}$, while $\mathrm{C}_{\alpha}$ - $\mathrm{C}$ bonds has two minimal energies if its bond rotation angle $\psi$ is $160^{\circ}$ and $-48^{\circ}$. The rotation angles $\varphi=-160^{\circ}$ and $\psi=160^{\circ}$ are assigned as trans $(t)$, and $\varphi=-48^{\circ}$ and $\psi=-73^{\circ}$ are assigned as gauche $(g)$. Accordingly, there are four conformations leading to four energy minima: $t t, t g, g t$, and $g g$, which correspond to $2_{1}, 5_{1}$, $10_{3}$, and $4_{1}$ helices [38]. 


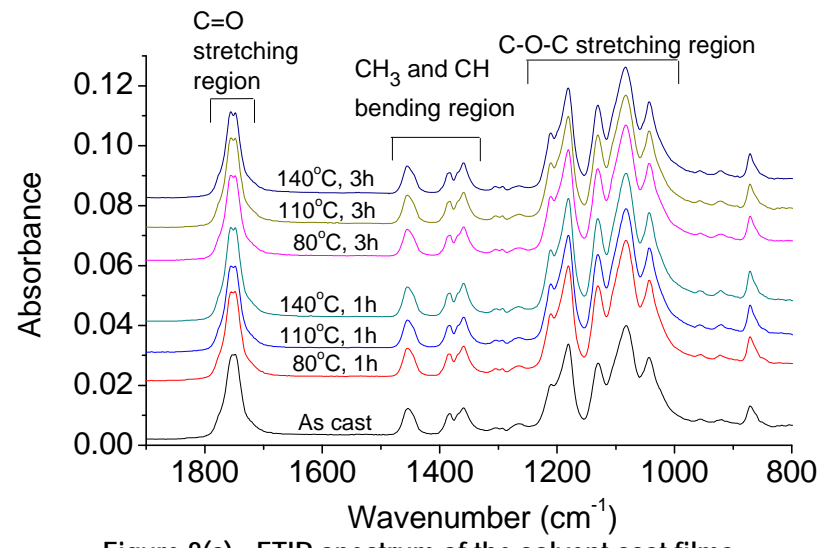

Figure 8(a) - FTIR spectrum of the solvent cast films.

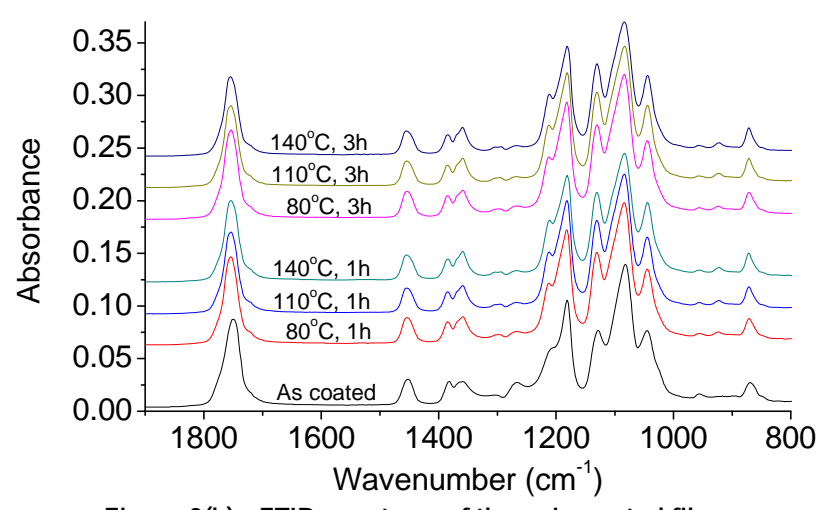

Figure 8(b) - FTIR spectrum of the spin coated films.

The gt conformation has the lowest energy and constitutes the crystal structure. Because of the four conformations, the carbonyl region can be split into four peaks due to intramolecular and intermolecular interactions, and the four peaks are located at $1776 \mathrm{~cm}^{-1}, 1767 \mathrm{~cm}^{-1}, 1759 \mathrm{~cm}^{-1}$, and 1749 $\mathrm{cm}^{-1}$, corresponding to $\mathrm{gg}, \mathrm{tg}, \mathrm{gt}$, and $\mathrm{tt}$ conformations, respectively [37]. The spectra of the $\mathrm{C}=\mathrm{O}$ stretching region of solvent cast and spin coated films, before and after annealing, are given in Figures 9(a), 9(b), and 10. It can be seen that, for the solvent cast films, annealing makes more prominent band splitting. However, band splitting is hard to be visually observed for the spin coated films.

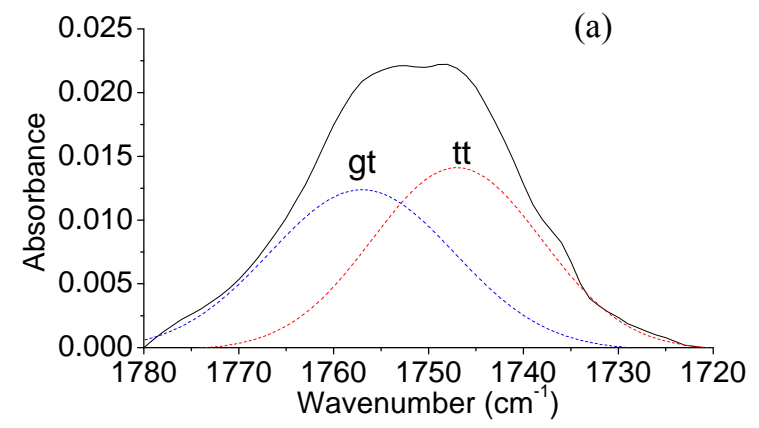

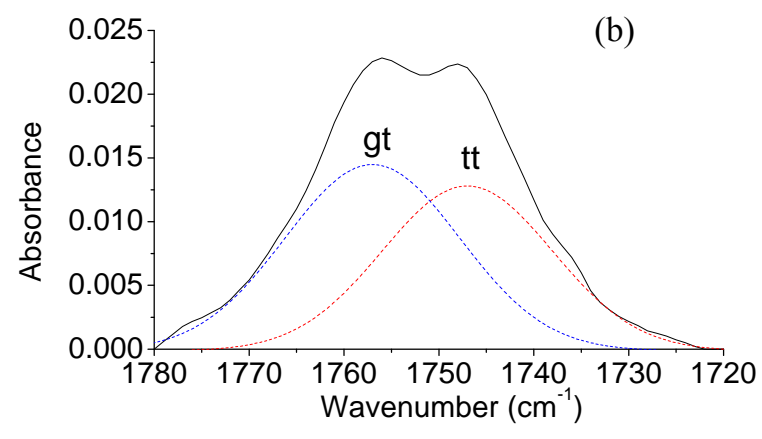

Figure 9 - Curve fitting for the $g t$ and $t t$ conformers in the carbonyl $\mathrm{C}=0$ stretching region of the solvent cast film (a) non-annealed and (b) annealed at $140^{\circ} \mathrm{C}$ for 3 hours.

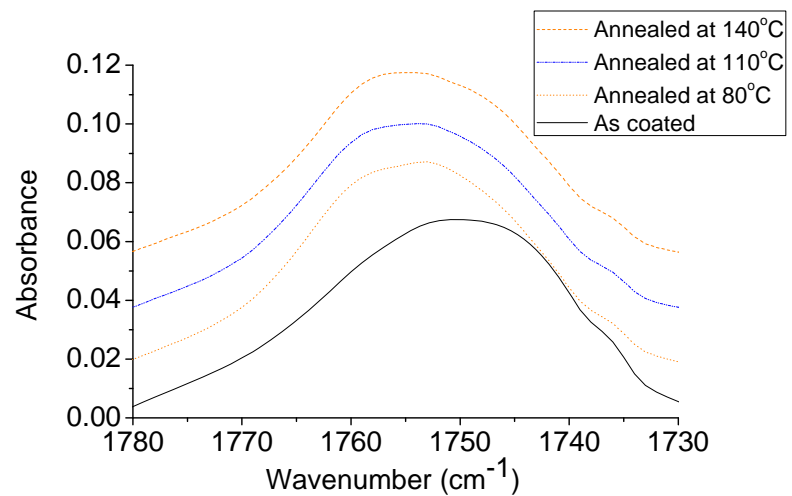

Figure 10 - Carbonyl $\mathrm{C}=0$ stretching region of the spin coated films annealed at different temperatures for 3 hours.

To investigate the components in the spectrum, the second derivatives of both films in different annealing conditions are determined and illustrated in Figure 11. For solvent cast films, two prominent peaks can be seen at $1757 \mathrm{~cm}^{-1}$ and 1747 $\mathrm{cm}^{-1}$, while two inconspicuous peaks are shown at $1777 \mathrm{~cm}^{-1}$ and $1766 \mathrm{~cm}^{-1}$. These four peaks can be attributed to the $\mathrm{gt}$, $t$, gg, and $t g$ conformations, respectively, and the most prominent two peaks, $g t$ and $t t$ are resolved, as given in Figures 9(a) and 9(b), respectively. It can be seen that the intensity ratio of $g t / t t$ increases as the film is annealed with its degree of crystallinity increasing. This confirms that the crystal structure obtained is preferred by the $g t$ conformation.

The peak width (defined as the full width at half maximum, FWHM) of the resolved peaks is shown in Figure 12. It is observed that, as the films are annealed, the width of the $g t$ peaks decreases, while that of the $t t$ peaks almost does not alter. The peak width of the FTIR spectrum is related to the homogeneity in the structure: more chemical environments lead to more complicated intermolecular interactions, which broadens the infrared bands; on the other hand, for molecules with weak intermolecular interactions and few chemical environments, the infrared bands are narrow [39]. Accordingly, since gt peak is responsible for the crystal structure, the narrowing $g t$ peaks suggest that as the structure gets crystallized, the crystal structure becomes more ordered, 
which leads to a more uniform and homogeneous chemical environment. On the contrary, the band width of the $t t$ conformation does not change significantly. This can be due to the fact that the $t t$ conformers do not contribute to crystallization, and remain amorphous after annealing.

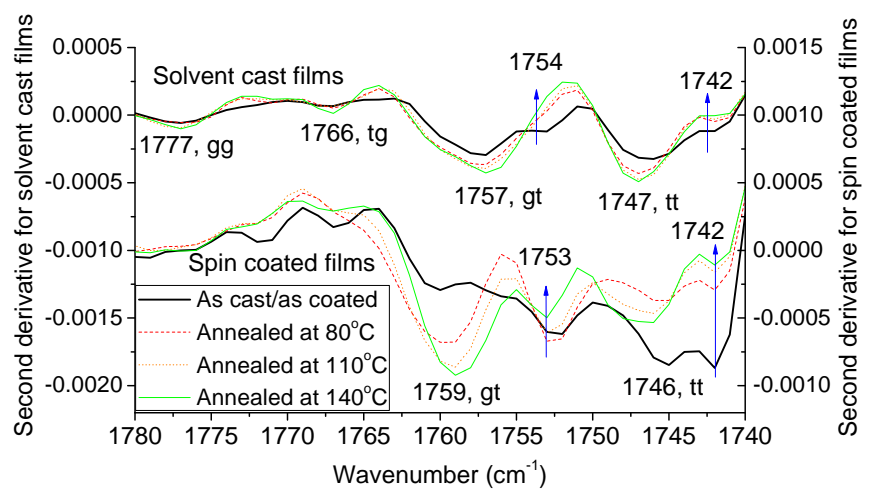

Figure 11 - Second derivative spectra of the $\mathrm{C}=0$ stretching region for solvent cast and spin coated films before annealing and annealed at different temperatures for 3 hours.

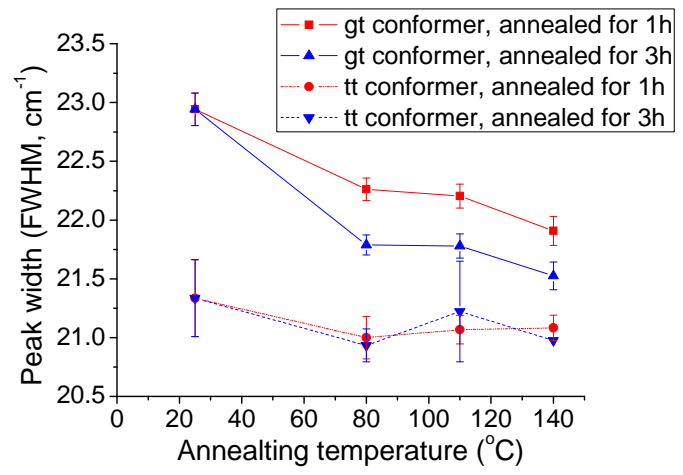

Figure 12 - The peak width of the resolved spectra for the gt conformer and tt conformer of the solvent cast films before annealing and after annealed in different conditions. The error bar represents the standard deviation of the five measurements.

It is also observed that there are two shoulders appearing at $1754 \mathrm{~cm}^{-1}$ and $1742 \mathrm{~cm}^{-1}$ for the non-annealed solvent cast film (Figure 11). The two shoulders cannot be attributed to any of the four conformers, and it is likely that they represent two peaks splitting from the $g t$ peak and $t t$ peak. To understand the origin of the shoulders, it is worth noting that they both split toward the lower wavenumbers. Stress is therefore a possible cause, in that it can increase the length of a covalent bond, which decreases the bond's atomic force constant, and thus decreases the infrared absorption frequency of bonds for their stretching vibrations [40]. The stress in the non-annealed solvent cast film may come from the nonuniformity of volume shrinkage during crystallization. After annealing, the 1754 $\mathrm{cm}^{-1}$ shoulder disappears, and the one at $1742 \mathrm{~cm}^{-1}$ also becomes less prominent, suggesting that annealing eliminates the stress in the structure.
For the spin coated films, the two prominent peaks are $g t$ and $t$, located at $1759 \mathrm{~cm}^{-1}$ and $1746 \mathrm{~cm}^{-1}$, respectively, while $g g$ and $t g$ peaks can hardly be attributed. There are two additional peaks shown at $1753 \mathrm{~cm}^{-1}$ and $1742 \mathrm{~cm}^{-1}$, which are also located on the right to the $g t$ and $t t$ peaks, respectively. The situation is similar to the solvent cast films, and it is most likely the peaks are again caused by the stress in the structure. In this case, the stress is due to the shear stress caused by the centrifugal force during spinning [35]. As the film is annealed, the peaks tend to become less prominent, suggesting the decrease of stress. It can also be seen that, in the derivative spectrum for the as coated film, the $t t$ peak is much more prominent than the gt peak, which is consistent with the fact that this film is amorphous, and may also explain why the band shifts to lower wavenumber in the original spectrum (Figure 10).

\section{The effect of annealing time}

The spin coated films are also annealed at $140^{\circ} \mathrm{C}$ for 8 hours and 24 hours. It is observed through optical microscopy that for longer annealing time, the boundaries can be seen more clearly, and the number of the eye structures is reduced, as shown in Figure 13. This suggests that more crystallites are formed at longer annealing time and that these lamellae gradually fill the space in the eye structures [30].

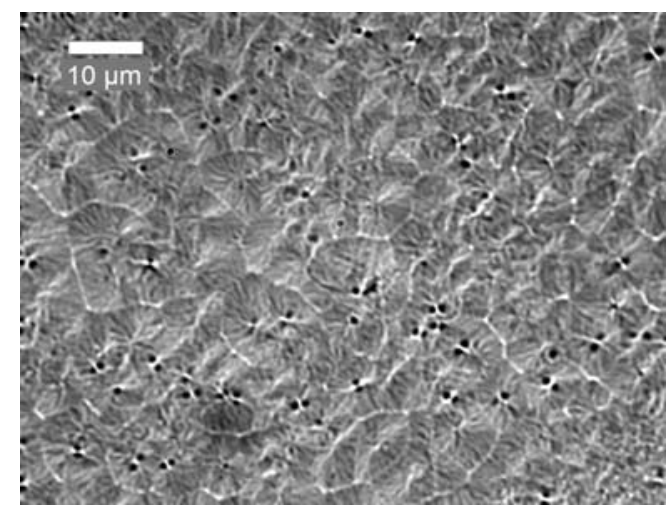

Figure 13 - The surface morphology of the thick spin coated film annealed at $140^{\circ} \mathrm{C}$ for 24 hours. The eye structure remains while the boundary of spherulites can be observed.

The WAXD results for the spin coated films annealed at $140^{\circ} \mathrm{C}$ for 24 hours are given in Figure 14 . Note that the $(010)$ peak emerges for both 8-hour annealing (not shown) and 24annealing. A possible reason is that during longer annealing time the nodules can be better aligned and create enough atomic planes for reflection to occur. Figure 15 gives the WAXD results of the degree of crystallinity and peak location; both increase with annealing time. The increase of degree of crystallinity is as expected, while the increase of the $2 \theta$ value suggests the crystals being more closely compacted. A more compact structure probably results from further disentanglement of polymer chains which leads to a more regular folding surface, so that the effect of folds is less prominent. It can also be due to the reduction of stress in the structure and change of conformation owing to the longer 
annealing time. The diagram also shows that the increasing trends tend to level off, which suggests that the crystal structure in the 24-hour annealed film is close to a stable structure and less likely to further change under even longer annealing time. It is thus implied that crystal structure (category 1 spherulites or category 2 spherulites) is determined at the early stage of their growth, and can hardly change once it is formed.

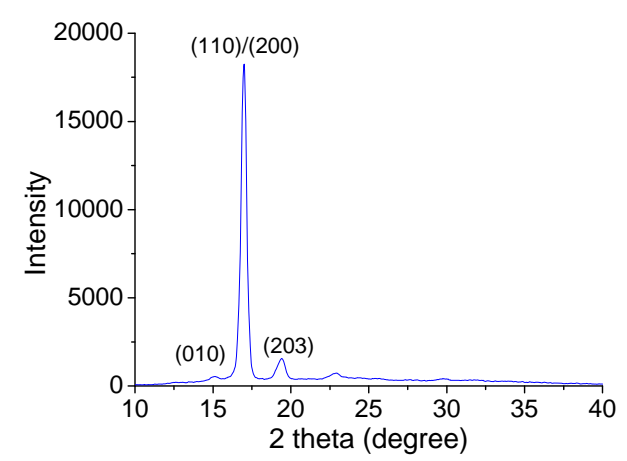

Figure 14 - The WAXD profile for the spin coated film annealed at $140^{\circ} \mathrm{C}$ for 24 hours. The (010) peak emerges.

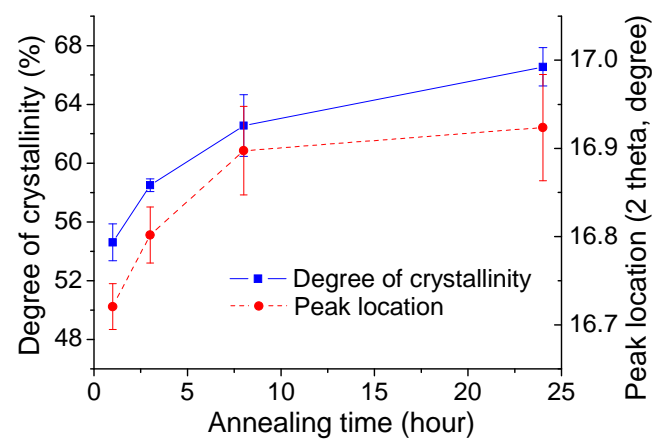

Figure 15 - The degree of crystallinity and peak location of the spin coated film annealed at $140^{\circ} \mathrm{C}$ for different amount of time: $1,3,8$, and 24 hours.

\section{CONCLUSIONS}

In this study, both the film formation method and annealing have been shown to have significant influences on PLLA crystallinity. In solvent cast films, category 1 spherulites are developed in solution during the casting process, and further annealing increases the degree of crystallinity without affecting morphology. Spin coating leads to an amorphous structure, while subsequent annealing generates category 2 spherulites. In addition to morphology, crystal structures in the two kinds of films are different based on WAXD measurements. The lamellae in the solvent cast film could be homogeneous, while those in the spin coated films may contain the nodular structure. The FTIR measurements reveal the conformation of the crystallites, and also shows possible stress in the films, which is reduced or eliminated through annealing. Longer annealing time stabilizes the development of category 2 spherulites, while their structures are similar to those obtained in shorter annealing time. This suggests that film formation method plays an important role on the resulting annealed crystal structures.

\section{ACKNOWLEDGMENTS}

Financial support from NSF under CMMI-1030536 is acknowledged. WAXD measurement at MRSEC, Columbia University is appreciated. Thanks are also due to Mr. Gen Satoh of Columbia University for helpful discussions.

\section{REFERENCES}

[1] Alexis, F., 2005, "Factors affecting the degradation and drug-release mechanism of poly(lactic acid) and poly[(lactic acid)-co-(glycolic acid)]," Polym. Int., 54, pp. 36-46

[2] Tsuji, H., Ikada, Y., 1998, "Properties and morphology of poly(L-lactide). II. Hydrolysis in alkaline solution," J. Polym. Sci. A: Polym. Chem., 36, pp. 59-66

[3] Tsuji, H., Ikada, Y., 1995, "Properties and morphology of poly(L-lactide). 1. Annealing condition effects on properties and morphologies of poly(L-lactide)," Polymer, 36(14), pp. 2709-2716

[4] Bhatla, A., Yao, Y. L., 2009, "Effect of Laser Surface Modification on the Crystallinity of Poly(L-Lactic Acid)," J. Manufacturing Science and Engineering, 131, pp. 051004-1051004-11

[5] Li, H., Nie, W., Deng, C., Chen, X., Ji, X., 2009, "Crystalline morphology of poly(L-lactic acid) thin films," Eur. Polym. J., 45, pp. 123-130

[6] Norton, D. R., Keller, A., 1985, "The spherulitic and lamellar morphology of melt-crystallized isotactic polypropylene," Polymer, 26, pp. 704-716

[7] Takeda, H., Nakashima, C., Nasu, N., 2005, "Studies on structure of poly( $\varepsilon$-L-lysine) spherulite grown from solution," Sen'I Gakkaishi, 61, pp. 61-66

[8] Wang, Z., Alfonso, G. C., Hu, Z., Zhang, J., He, T., 2008, "Rhythmic growth-induced ring-banded spherulites with radial periodic variation of thicknesses grown from poly $(\varepsilon-$ caprolactone) solution with constant concentration," Macromolecules, 41, pp. 7584-7595

[9] Pluta, M., Galeski, A., 2002, "Crystalline and Supermolecular Structure of Polylactide in Relation to the Crystallization Method," J. Appl. Polym. Sci., 86, pp. 13861395

[10] Zhao, J., Qiu, J., Niu, Y., Wang, Z., 2009, "Evolutions of Morphology and Crystalline Ordering Upon Annealing of Quenched Isotactic Polypropylene," J. Polym. Sci. B, 47, pp. 1703-1712

[11] Su, C. H., Jeng, U., Chen, S. H., Lin, S. J., Wu, W. R., Chuang, W.-T., Tsai, J. C., Su, A. C., 2009, "Nanograin evolution in cold crystallization of syndiotactic polystyrene as illustrated via in-situ small/wide angle X-ray scattering and differential scanning calorimetry," Macromolecules, 42, pp. 6656-6664 
[12] Hsu, C. C., Geil, P. H., Miyaji, H., Asai, K., 1986, "Structure and Properties of Polypropylene Crystallized from the Glassy State,” J. Polym. Sci. B, 24, pp. 2379-2401

[13] Ogawa, T., Miyaji, H., Asai, K., 1985, "Nodule structure of polypropylene," J. Phys. Soc. Japan, 54(10), pp. 3668-3670

[14] Bassett, D. C., Olley, R. H., Al Raheil, I. A. M., 1988, "On crystallization phenomena in PEEK," Polymer, 29, pp. 17451754

[15] Ivanov, D. A., Jonas, A. M., 1998, "Isothermal growth and reorganization upon heating of a single poly(aryl-ether-etherketone) (PEEK) spherulite, as imaged by atomic force microscopy," Macromolecules, 31, pp. 4546-4550

[16] Kobayashi, J., Asahi, T., Ichiki, M., Oikawa, A., Suzuki, H., Watanabe, T., Fukada, E., Shikinami, Y., 1995, "Structural and optical properties of poly lactic acids," J. Appl. Phys. 77(7), pp. 2957-2973

[17] Miyata, T., Masuko, T., 1997, "Morphology of poly(Llactide) solution-grown crystals," Polymer, 38(16), pp. 40034009

[18] Sasaki, S., Asakura, T., 2003, "Helix distortion and crystal structure of the $\alpha$-form of poly(L-lactide)," Macromolecule, 36, pp. 8385-8390

[19] Brillart, M. V., Cebe, P., 1995, "Thermal expansion of the crystal lattice of novel thermoplastic polyimides," J. Polym. Sci. B, 33, pp. 927-936

[20] Hay, J. N., Langford, J. I., Lloyd, J. R., 1989, "Variation in unit cell parameters of aromatic polymers with crystallization temperature" Polymer, 30, pp. 489-493

[21] Davis, G. T., Eby, R. K., Martin, G. M., 1968, "Variations of the unit-cell dimensions of polyethylene: effect of crystallization conditions, annealing, and deformation," J. Appl. Phys., 39(11), pp. 4973-4981

[22] Zhang, A., Jiang, H., Wu, Z., Wu, C., Qian, B., 1991, "Internal Stress, Lattice Deformation, and Modulus of Polymers,” J. Appl. Polym. Sci., 42, pp. 1779-1791

[23] Kikkawa, Y., Abe, H., Iwata, T., Inoue, Y., Doi, Y., 2002, "Crystallization, stability, and enzymatic degradation of poly(L-lactide) thin film," Biomacromolecules, 3(2), pp. 350356

[24] Schultz, J. M., 2001, Polymer Crystallization: The Development of Crystalline Order in Thermoplastic Polymers, Oxford University Press, New York

[25] Hoffman, J. D., Davis, G. T., Lauritzen, J. I., 1976, in Treatise on Solid State Chemistry, vol. 3, Hanny, N. B., Ed., Plenum Press, New York

[26] Strobl, G., 2000, "From the melt via mesomorphic and granular crystalline layers to lamellar crystallites: A major route followed in polymer crystallization?” Eur. Phys. J. E, 3, pp. $165-183$
[27] Grasruck, M., Strobl, G., 2003, "Crystallization of spolypropylene from the glassy state: indications for a multistage process," Macromolecules, 36, pp. 86-91

[28] Menczel, J., Wunderlich, B., 1981, "Heat capacity hysteresis of semicrystalline macromolecular glasses," J. Polym. Sci.: Polym. Lett. Ed., 19, pp. 261-264

[29] Granasy, L., Pusztai, T., Tegze, G., Warren, J. A., Douglas, J. F., 2005, "Growth and form of spherulites," Phys. Rev. E, 72, pp. 011605-1-011605-15

[30] Li, L., Chan, C.-M., Yeung, K. L., Li, J.-X., Ng, K.-M., Lei, Y., 2001, "Direct observation of growth of lamellae and spherulites of a semicrystalline polymer by AFM," Macromolecules, 34, pp. 316-325

[31] Frank, C. W., Rao, V., Despotopoulou, M. M., Pease, R. F. W., Hinsberg, W. D., Miller, R. D., Rabolt, J. F., 1996, "Structure in thin and ultrathin spin-cast polymer films," Science, 273, pp. 912-915

[32] Cotton, J. P., Nierlich, M., Boue, F., Daoud, M., Farnoux, B., Jannink, G., Duplessix, R., and Picot, C., 1976, "Experimental Determination of the TemperatureConcentration Diagram of Flexible Polymer Solutions by Neutron Scattering," J. Chem. Phys., 65(3), pp. 1101-1108.

[33] Toda, A., Taguchi, K., Kajioka, H., 2008, "Instabilitydriven branching of lamellar crystals in polyethylene spherulites," Macromolecules, 41, pp. 7505-7512

[34] Wunderlich, B., 1976, Macromolecular Physics, Volume 2, Crystal Nucleation, Growth, Annealing, Academic Press, New York

[35] Vorotilov, K., Petrovsky, V., Vasiljev, V., 1995, "Spin coating process of sol-gel silicate films deposition: effect of spin speed and processing temperature," J. Sol-Gel Sci. and Tech., 5, pp. 173-183

[36] Keith, H. D., Padden, F. J., Jr., 1963, "A phenomenological theory of spherulitic crystallization," J. Appl. Phys., 34(8), pp. 2409-2421

[37] Meaurio, E., Zuza, E., Lopez-Rodriguez, N., Sarasua, J. R., 2006, "Conformational behavior of poly(l-lactide) studied by infrared spectroscopy," J. Phys. Chem., 110, pp. 5790-5800

[38] Kang, S., Hsu, S. L., Stidham, H. D., Smith, P. B., Leugers, M. A., Yang, X., 2001, "A spectroscopic analysis of poly(lactic acid) structure," Macromolecules, 34, pp. 45424548

[39] Smith, B., 1999, Infrared Spectral Interpretation: A Systematic Approach, CRC Press, Boca Raton, FL

[40] Roylance, D. K., DeVries, K. L., 1971, "Determination of atomic stress distribution in oriented polypropylene by infrared spectroscopy," Polym. Lett., 9, pp. 443-447 